\title{
A MAUT APPROACH FOR REUSING DOMAIN ONTOLOGIES ON THE BASIS OF THE NeOn METHODOLOGY
}

\author{
A. JIMÉNEZ,**, M. C. SUÁREZ-FIGUEROA ${ }^{\dagger, \S}$, A. MATEOS ${ }^{*, \uparrow}$ \\ and A. GÓMEZ-PÉREZ ${ }^{\dagger, \|}$ \\ ${ }^{*}$ Decision Analysis and Statistics Group, \\ Dept. de Inteligencia Artificial, \\ Universidad Politécnica de Madrid, Boadilla del Monte, \\ Madrid 28660, Spain \\ ${ }^{\dagger}$ Ontology Engineering Group, \\ Dept. de Inteligencia Artificial, \\ Universidad Politécnica de Madrid, Boadilla del Monte, \\ Madrid 28660, Spain \\ 末ajimenez@fi.upm.es \\ $\S_{\text {mcsuarez@fi.upm.es }}$ \\ Tamateos@fi.upm.es

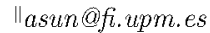

\section{FERNÁNDEZ-LÓPEZ} \\ Escuela Politécnica Superior, Universidad San Pablo CEU, \\ Boadilla del Monte, Madrid 28660 , Spain \\ mfernandez.eps@ceu.es
}

Knowledge resource reuse is becoming a widespread approach in the ontology engineering field because it can speed up the ontology development process. In this context, the $\mathrm{NeOn}$ Methodology specifies some guidelines for reusing different types of knowledge resources (ontologies, nonontological resources, and ontology design patterns). These guidelines prescribe how to perform the different activities involved in any of the diverse types of reuse processes. One such activity is to select the best knowledge resources for reuse in an ontology development. This selection activity is a complex decision-making problem involving conflicting objectives, like understandability, integration or reliability. We propose a multiattribute utility theory (MAUT) approach to deal with the selection of the best domain ontologies for reuse, stressing the identification of attributes to measure ontology performances. We take advantage of the sensitivity analysis tools provided by the GMAA system, a PC-based decision support system based on an additive multi-attribute utility model, to exploit imprecise information on the inputs. An example concerning the selection of a subset of ontologies for reuse in the development of a new ontology in the sports domain illustrates the approach. 


\section{Introduction}

Ontologies are formalized vocabularies of terms that cover a specific domain of interest and are shared by a community of users. ${ }^{a}$ One of the better known definitions follows: "An ontology is a formal, explicit specification of a shared conceptualization. Conceptualization refers to an abstract model of some phenomenon in the world by having identified the relevant concepts of that phenomenon. Explicit means that the type of concepts used, and the constraints on their use are explicitly defined. Formal refers to the fact that the ontology should be machine-readable. Shared reflects the notion that an ontology captures consensual knowledge, that is, it is not private of some individual, but accepted by a group". ${ }^{1}$ A great many of ontologies have been developed to date by different groups, taking different approaches and using different methodologies, methods and techniques. A series of methods and methodologies for developing ontologies from scratch have been reported in Ref. 2. As opposed to custom-building new ontologies from scratch, a new approach is now being followed in the ontology engineering field, ${ }^{3}$ emphasizing: (1) knowledge resource (ontologies, nonontological resources, and ontology design patterns) reuse and subsequent reengineering, (2) collaborative and argumentative ontology development, and (3) construction of ontology networks. ${ }^{b}$ If applied efficiently, this approach is able to reduce the time and costs associated with ontology development, because it avoids the re-implementation of existing ontologies. Additionally, this approach has the potential to (a) spread good practices and (b) increase the overall quality of ontological models.

Different approaches have been proposed to support the ontology reuse process: Fernández-López and colleagues ${ }^{5}$ and Uschold and colleagues ${ }^{6}$ propose different activities and tasks for reusing domain ontologies. Pinto and Martins ${ }^{7}$ propose some activities for reusing ontologies as part of the integration process. Finally, Paslaru and $\mathrm{Mochol}^{8}$ propose an incremental reuse process.

Most of the above methods recommend high-level steps but do not provide detailed guidelines explaining how to perform each step. However, the NeOn Methodology ${ }^{3,9}$ sets out some prescriptive methodological guidelines for reusing general and domain ontologies. These guidelines cover the following activities: (1) search repositories and registries for candidate ontologies that could satisfy the needs of the ontology network under development; (2) assess whether the set of candidate ontologies are useful for building the ontology network; (3) select the best candidate ontologies for developing the ontology network on the basis of a set of criteria; and, (4) integrate the selected ontologies into the ontology network under construction. Note that the NeOn Methodology is one of the main outcomes of the NeOn project

\footnotetext{
${ }^{a}$ http://www.w3.org/TR/owl2-overview/.

${ }^{b}$ An ontology network or a network of ontologies is defined as a collection of ontologies (called networked ontologies) related together through a variety of different meta-relationships such as mapping, modularization, version, and dependency relationships (Ref. 4).
} 
(Life Cycle Support for Networked Ontologies), which was funded by the European Commission's Sixth Framework Programme under grant number FP6-027595.

The NeOn Methodology is a scenario-based methodology that provides guidance for all main processes and activities in ontology engineering projects. The ONTO$C O M$ model $^{\mathrm{c}}$ provides information about how many people should be involved in a particular ontology network development. This is a cost estimation model, whose goal is to predict the costs (expressed in person months) of typical ontology engineering processes and activities.

Additionally, several researchers have already suggested series of criteria for ranking and selecting ontologies. Pinto and Martins, ${ }^{10}$ for example, focus on a set of criteria related to what knowledge is missing, what knowledge is superfluous; what knowledge should be relocated, and which knowledge sources, documentation, terminology, definitions, and practices should be changed. Lozano-Tello and GómezPérez ${ }^{11}$ define a detailed set of 117 criteria, organized in a three-level framework, for selecting the best ontology. Alani and Brewster ${ }^{12}$ propose AKTiveRank, a system for ranking ontologies that aggregates several measures related to structural features of ontology concepts. The measures used in AKTiveRank are ontology coverage for given terms, concept centrality in a hierarchy and in the ontology, structural density, and semantic similarity between concepts. Park and colleagues ${ }^{13}$ present OntologyRank, an approach for selecting and ranking ontologies based on semantic matching as well as lexical matching. The total score is calculated by aggregating the values from three-criteria measures (semantic similarity, topic coverage, and richness). Each measure has a weight that depends on the domain and context, thus they use the regression model to fit such weights. Finally, Martínez-Romero and colleagues $^{14}$ propose an approach for automatic ontology recommendation. This approach is based on measuring the adequacy of an ontology to a given context according to three independent criteria: (a) the coverage of the ontology in the requested context, (b) the semantic richness of the ontology in the context, and (c) the popularity of the ontology.

All the above criteria focus mainly on analyzing particular ontology viewpoints (knowledge features in Ref. 10; structural features in AKTiveRank, and semantic and lexical issues in OntologyRank), but they do not normally combine different dimensions like coverage, semantic richness and reliability as in Ref. 14 or the complex framework proposed in Ref. 11. For these reasons, a manageable collection of criteria and their associated measurement attributes that together consider different dimensions is needed to help ontology developers choose the best ontologies for reuse in a new ontology development.

Thus, the selection of the best ontologies for reuse in the development of a new ontology is a complex decision-making problem where different conflicting objectives have to be taken into account simultaneously. Several multicriteria decision analysis methodologies could be used to tackle this selection problem. We propose using the 
GMAA decision support system to facilitate this selection task. GMAA is based on multi-attribute utility theory (MAUT).

Section 2 summarizes the methodological guidelines proposed in the $\mathrm{NeOn}$ Methodology for reusing domain ontologies in the development of a new ontology. In Sec. 3, multicriteria decision analysis approaches for reusing ontologies are overviewed. In Sec. 4, we propose a MAUT approach for selecting domain ontologies for reuse in the development of a new ontology network. We illustrate the proposed approach for selecting the best sports ontologies for reuse in the development of a new ontology in the sports domain. Finally, some conclusions and future research lines are provided in Sec. 5 .

\section{Guidelines for Reusing Domain Ontologies in the NeOn Methodology}

The goal of reusing domain ontologies is to find and select one or more domain ontologies that can be reused to develop a new ontology. In accordance with the methodological guidelines proposed in the NeOn Methodology, this reuse process is composed of four activities, ${ }^{3,9}$ which are explained in detail as follows.

Activity 1. Domain Ontology Search. The objective of this activity is to search libraries, repositories, and registries for candidate domain ontologies that could satisfy the needs of the ontology network under development. The terms specified in the pre-glossary included in the ontology requirements specification document $(\mathrm{ORSD})^{15}$ are the input for this activity.

Activity 2. Domain Ontology Assessment. The objective of this activity is to find out if the set of candidate domain ontologies are useful for developing the ontology network. The input for this activity is the set of domain ontologies output in Activity 1. In this activity, the ontology development team should remove the domain ontologies that are unsuitable for reuse from the set.

To carry out this activity, the methodological guidelines recommend analyzing the domain coverage in order to decide whether a particular domain ontology is useful. This means studying whether the domain ontology totally or partially covers the requirements identified in the ORSD of the ontology network that is being developed. To do this, the methodological guidelines propose the following actions:

- Check whether the purpose established in the ORSD and the purpose of the candidate domain ontology are similar. The results of this informal checking can be 'Yes' if both purposes are similar (e.g., when the purpose of both ontologies is to provide a consensual knowledge model of the employment domain that can be used by public e-Employment services), 'No' if both purposes are not comparable (e.g., when the candidate ontology was conceived for use for job searching purposes and the new ontology is going to be used for gathering employment statistics), or 'Unknown' if information about the purpose of the candidate ontology is unavailable. 
- Check whether the scope established in the ORSD and the scope of the candidate domain ontology are similar. The results of this informal checking can be 'Yes' if both scopes are similar (e.g., when both ontologies have to represent knowledge related to employment issues in the ICT (information and communication technology) domain), 'No' if both scopes are not comparable (e.g., when the candidate ontology represents employment issues in the agriculture domain and the new ontology is to represent employment issues in the ICT domain), or 'Unknown' if information about the scope of the candidate ontology is unavailable.

- Check whether nonfunctional ontology requirements established in the ORSD are covered by the candidate domain ontology. Examples of nonfunctional requirements can be "terms to be used in the ontology must be taken from standards", "multilinguality must be represented in the ontology to be developed", etc. This checking should be performed manually by informally comparing the set of nonfunctional requirements.

- Check whether the candidate domain ontology totally or partially covers functional requirements in the form of competency questions (CQs) listed in the ORSD. These checks can be run in three different ways:

- Partially, by analyzing if the essential terms for the new ontology development appear in the candidate domain ontology to be reused. ${ }^{16,17}$

- Again partially, by calculating the precision and coverage of the terminology of the candidate domain ontologies with respect to the terminology included in CQs.

* Precision ${ }^{18}$ is defined as the proportion of the retrieved material that is actually relevant. To adapt this measure to the ontological context, it is necessary to define CandidateDomainOntologyTerminology as the set of terms included in the candidate domain ontology and ORSDTerminology as the set of identified terms included in the ORSD. Thus, within the ontological context, precision is defined as the ratio of candidate domain ontology terms listed in the terms identified in the ORSD to candidate domain ontology terms.

* Coverage is based on the recall measure used in information retrieval. ${ }^{18}$ Recall is defined as the proportion of relevant material actually retrieved to answer a search request. To adapt this measure to the ontological context, the above mentioned definitions of CandidateDomainOntologyTerminology and ORSDTerminology are used. Thus, in this context, coverage is the ratio of the terms identified in the ORSD that are listed in the candidate domain ontology terms to terms identified in the ORSD.

- Totally, by determining whether the candidate domain ontology is able to answer the CQs listed in the ORSD.

The ontology development team analyzes the set of candidate domain ontologies according to the above-mentioned criteria. As an output of this analysis, the team should fill in an assessment table modeled on the template shown in Table 1. 
Table 1. Assessment table template.

\begin{tabular}{|c|c|c|}
\hline Criteria & Range of values & $\begin{array}{c}\text { Candidate domain ontologies } \\
\text { ontology1 ontology } 2 \ldots \text { ontologyn }\end{array}$ \\
\hline Similar purpose & [Yes, No, Unknown] & \\
\hline Similar scope & [Yes, No, Unknown] & \\
\hline $\begin{array}{l}\text { Nonfunctional requirements } \\
\text { coverage }\end{array}$ & $\begin{array}{l}\text { [Yes-Totally, Yes-Partially, } \\
\text { No, Unknown] }\end{array}$ & \\
\hline $\begin{array}{l}\text { Functional requirements } \\
\text { coverage }\end{array}$ & $\begin{array}{c}\text { [Yes-Totally, Yes-Partially, } \\
\text { No, Unknown] }\end{array}$ & \\
\hline
\end{tabular}

The heuristic used to reject a candidate domain ontology is: if the ontology developer has answered No to the Similar Scope and/or Similar Purpose and/or Functional Requirements coverage criteria for a particular candidate domain ontology, then the candidate ontology should be considered not useful and should be removed from the set of candidate ontologies.

Activity 3. Domain Ontology Selection. The objective of this activity is to find out which domain ontologies are the most suitable for the development of the ontology network. The input for this activity is the set of domain ontologies obtained in Activity 2 . To identify the most suitable candidate domain ontologies, we propose to use the updated set of criteria and attributes presented in Sec. 4.1.

Activity 4. Domain Ontology Integration. The objective of this activity is to integrate the domain ontologies selected in Activity 3 into the ontology under development.

\section{MCDA Approaches for Selecting Ontologies}

As mentioned in Sec. 1, the selection of the most suitable domain ontologies for reuse in the development of a new ontology is a complex decision-making problem where different conflicting objectives, like understandability effort, integration effort, and reliability, have to be taken into account simultaneously.

Different multicriteria decision analysis (MCDA) methodologies, such as MAUT, ${ }^{19-21}$ AHP,${ }^{22,23}$ or outranking methods, e.g., ELECTRE ${ }^{24}$ and PROMETHEE ${ }^{25,26}$ could be used to tackle this selection problem.

Although ontology ranking was traditionally based on a weighted sum of several quality measures (Refs. 13 and 27-28), decision maker (DM) preferences have more recently been incorporated into the analysis. In this respect, Lozano-Tello and Gómez-Pérez introduced a new method, ONTOMETRIC, for choosing the best ontology based on AHP methodology in Ref. 11; while Esposito et al. compared two ELECTRE methods with AKTiveRank in Ref. 29, demonstrating their suitability for ontology ranking.

There is no agreement about which is the best methodology for all decisionmaking situations. As it is hard to determine which aspects should be considered to select an appropriate approach, see Ref. 30, the decision usually depends on the problem in question, the model that decision makers are most comfortable and 
familiar with or like most. According to Linkovet al., ${ }^{31}$ selecting an approach from the available methods may be itself an expression of subjective values or a purely pragmatic choice (such as familiarity or perceived ease of implementation).

The findings concerning experiences gathered about the application of MCDA methods over the years to various domains and cases follow:

- Outranking methods do not always take into account whether over-performance in one criterion can make up for under-performance in another, see Ref. 32 .

- In the mid-1980s, MCDA researchers began to compare the AHP to MAUT. They found that AHP does not adhere to the basic von Neumann and Morgenstern axiomatic structure of normative utility theory as incorporated in MAUT, and they raised other concerns. For example, Dyer ${ }^{33}$ reviewed the criticism of the AHP and concluded that the AHP is "flawed", and Smith and von Winterfeldt" reviewed past Management Science papers on decision analysis and stated: "...many in the decision analysis community (ourselves included) follow Dyer in believing the AHP to be fundamentally unsound,..."

- Wang et al. compare MAUT and PROMETHEE and reach the conclusion that "MAUT takes any difference in any criterion into account and is able to provide completely preference order of all alternatives simultaneously. The impacts of any changes in values can be revealed by sensitivity analysis". ${ }^{35}$

In view of the above findings and MCDA guidelines, we have decided to use MAUT-based decision analysis methodology taking into account that we are experts on MAUT and that this methodology has not been applied until now for selecting ontologies for reuse.

Decision analysis (DA) is aimed at structuring and simplifying the task of making hard decisions as well and as easily as the type of decision permits. ${ }^{19,36-38} \mathrm{DA}$ is developed on the assumption that the alternatives will appeal to experts depending on how likely the possible performances of each alternative are and what preferences experts have for the possible performances.

What sets DA apart is how these factors are quantified and formally incorporated into the problem analysis. Existing information, collected data, models and professional judgments are used to quantify the likelihoods of a range of performances, and utility theory is used to quantify preferences. DA can be divided into four steps: (a) structure the problem; (b) identify feasible strategies, their impact and uncertainty (if necessary); (c) quantify preferences; and (d) evaluate alternatives and analyze sensitivity.

The Generic Multi-Attribute Analysis System $^{39,40}$ (GMAA) is a user-friendly PCbased decision support system that is intended to allay the operational difficulties involved in the DA methodology. The GMAA system ${ }^{\mathrm{d}}$ has been used by the authors to support different complex decision-making problems. ${ }^{41-43}$ It accounts for uncertainty ${ }^{44,45}$ about alternative performances and for incomplete information about DM 
preferences, which leads to classes of component utility functions and weight intervals. Moreover, it checks inconsistencies in DM responses when assessing their preferences.

The alternatives are evaluated by means of an additive multi-attribute utility function, which is used to assess, average overall utilities, on which the ranking of alternatives is based, and also, minimum and maximum overall utilities, which give further insight into the robustness of this ranking. As in most cases, the information obtained is not meaningful enough so as to definitively recommend an alternative (very overlapped utility intervals are output), the system provides several tools for sensitivity analysis that take advantage of the imprecise input to reach further insight into the robustness of the ranking of alternatives.

\section{A MAUT Approach for Selecting Ontologies}

In this section, we propose a MAUT approach for selecting domain ontologies for reuse in the development of a new ontology network. In this general approach, we use the GMAA system to support the selection.

As already mentioned in Sec. 2, the aim of the domain ontology selection activity is to find out the most appropriate domain ontologies for reusing in the development of a new ontology network. To identify the most suitable domain ontologies, several conflicting objectives have to be taken into account simultaneously. That is, the candidate ontologies should be analyzed with respect to a set of criteria and attributes. After such an analysis, a table with the ontology performances is obtained. The values in this table are then introduced in the GMAA system, which should be configured with the particular preferences depending on the situation (which attributes are most important and which nature have them). GMAA obtains a ranking of the candidate ontologies. The approach we propose is to select those domain ontologies that (a) have better score in the ranking and (b) cover the largest possible number of requirements, having the set of selected ontologies as minimal as possible. Thus, this approach involves a trade-off between the number of selected ontologies and the percentage of requirements covered by such ontologies.

We illustrate how the proposed approach and the GMAA system are used to select the best sports ontologies for reuse in the development of a new ontology in the sports domain.

\subsection{Criteria and corresponding attributes}

An initial collection of criteria to be taken into account for domain ontology reuse was explained in Ref. 3. The collection included 14 criteria organized in four dimensions (reuse cost, understandability effort, integration effort, and reliability). This initial set of criteria was modified in Refs. 46 and 47 . In this paper, we have manually analyzed both set of criteria and decided (a) to delete some of the criteria due to the difficulty of measuring them in an objective way (e.g., development team 


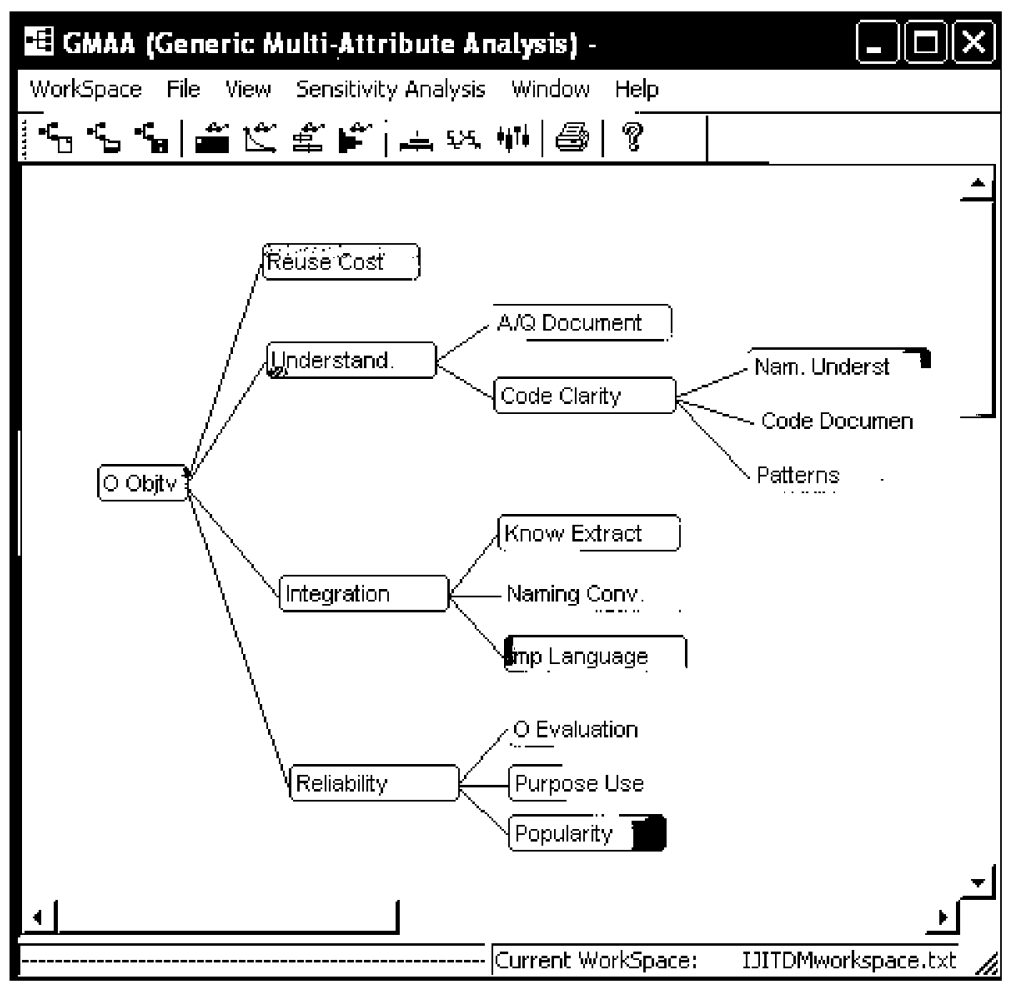

Fig. 1. Objective hierarchy.

reputation) and (b) to merge other criteria because they were slightly overlapped (e.g., availability of tests and former evaluation). As a result of this analysis, we have obtained a set of 11 criteria organized according to four main objectives, as shown in Fig. 1. In addition to the update in the quantity, we have improved the collection of criteria by means of defining associated attributes to measure criteria in an objective fashion.

Reuse cost refers to the financial cost of accessing and using the candidate ontology. A continuous attribute representing the possible cost (in euros or dollars) of the different ontology licenses is associated with this criterion. If the candidate ontology has a free license, then the value should be zero. Otherwise the value for this criterion should be the cost of purchasing or using the license.

Note that in the ontology engineering area most ontologies are currently available free of charge (that is, have a free license). However, the ontology engineering community is discussing licensing issues, and this criterion will probably have to be taken into account in the future when there are different types of ontology licenses.

Understandability effort (Understand.) is an estimate of the workload involved in understanding the candidate ontology. It has been split into two subcriteria, documentation availability and quality and code clarity. 
Documentation availability and quality (A/Q Document) refers to whether there is any communicable material used to describe or explain different aspects of the candidate ontology, as well as the enacted development process. The documentation should explain the statements contained in the ontology for a novice target audience. ${ }^{10} \mathrm{~A}$ discrete attribute with three possible values has been established to measure this criterion:

- low, when the candidate ontology has no documentation;

- medium, when the candidate ontology has documentation (in the form of web page, wiki, and/or paper) detailing the ontology; and

- high, when the candidate ontology has documentation (in the form of web page, wiki, and/or paper) detailing both the candidate ontology and its development process (ORSD, modeling decisions, etc.).

Code clarity refers to whether the code is easy to understand and modify, that is, if the knowledge entities follow unified patterns and are intuitive. ${ }^{10}$ It is advantageous to use the same pattern for sibling definitions, thus improving ontology understanding and making it easier to include new definitions. ${ }^{48}$ Clarity also refers to whether the code is well documented, that is, if it includes clear and coherent definitions and comments for the knowledge entities represented in the candidate ontology. The difference between this criterion and the documentation availability and quality is that code clarity refers to the element definitions and comments inside the ontology code, whereas the documentation availability and quality of the documentation refers to external documentation (papers, manuals, etc.).

Code clarity has been split into the following three subcriteria: naming understandability, code documentation and pattern conformance. Naming understandability (Nam. Underst) refers to the clarity of the ontology element names. A binary attribute has been associated with this subcriterion accounting for the name intuitiveness (0-not intuitive, 1-intuitive). Code documentation (Code Documen) refers to whether the ontology code is documented. A continuous scale within $[0,100]$ was used to measure this attribute, computed as follows:

$$
\text { \#CommentedOntologyElements } \times 100 / \# \text { OntologyElements, }
$$

where \#CommentedOntologyElements is the number of elements commented in the ontology (rated by searching the code for the "rdfs:comment" string) and \#OntologyElements is the number of elements in the ontology (\#OntologyElements $=1+$ \#Classes + \#ObjectProperties + \#DatatypeProperties + \#Instances).

Finally, pattern conformance (Patterns) refers to whether the ontology has been developed using ontology design patterns (ODPs). ${ }^{\text {e }}$ Three pattern types are used to measure this subcriterion: logical patterns $(L P)$, content patterns $(C P)$ and

ehttp://ontologydesignpatterns.org/. 
reasoning patterns $(R P)$. The other patterns listed in the ODP catalogue are not taken into account, because they cannot be directly located in the ontology. For each of the three pattern types, we check whether the ontology uses at least one pattern of each type, leading to the following discrete attribute values (no patterns, $L P, C P$, $R P, L P+C P, L P+R P, C P+R P, L P+C P+R P)$. For instance, $L P+R P$ means that the ontology uses at least one logical pattern and one reasoning pattern but it does not use any content pattern.

Integration effort (Integration) is an estimate of the workload for integrating the candidate ontology into the ontology under development. We consider the adequacy of knowledge extraction, the adequacy of naming conventions and the adequacy of the implementation language.

The adequacy of knowledge extraction (Know Extract) refers to whether it is easy to identify parts of the candidate ontology to be reused. This is basically concerned with whether a modular approach has been used to develop the candidate ontology, i.e., whether the ontology is composed of different modules. A binary attribute, indicating whether or not the ontology is modular (0-no, 1-yes), was used to measure this criterion. Note that there are tools for extracting modules if the ontology is not modularized.

The adequacy of naming conventions (Naming Conv) refers to whether the two ontologies (the candidate ontology and the ontology under development) follow the same rules for naming the different ontology components (e.g., one possibility is that concept names start with capital letters and relation names start with noncapital letters). This criterion can be measured using a binary attribute with value 1 when the candidate ontology and the ontology under development follow the same naming conventions and value 0 when they do not.

The adequacy of the implementation language (Imp Language) refers to whether the languages (of the candidate ontology and the ontology under development) are the same or are at least able to represent similar knowledge with the same granularity. A discrete attribute with three possible values was established to measure this criterion:

- low, when the transformation between the language of the candidate ontology and the language of the ontology to be developed is not easy;

- medium, when the transformation between the language of the candidate ontology and the language of the ontology to be developed is easy; and

- high, when the candidate ontology and the ontology to be developed are in the same language.

Finally, reliability refers to an analysis of whether ontology developers can trust the candidate ontology for reuse. Reliability is rated by ontology evaluation, purpose of use and popularity.

Ontology evaluation (O Evaluation) refers to whether the ontology has been properly evaluated. If there is no information on this issue, OOPS! ${ }^{\mathrm{f}}$ should be used to

$\mathrm{f}_{\text {http://www.oeg-upm.net/oops. }}$ 
evaluate the candidate ontologies in order to determine whether candidate ontologies contain errors or anomalies. OOPS! is a web application that helps ontology developers to detect some of the most common pitfalls in ontologies. ${ }^{49}$ OOPS! detects 21 common pitfalls and reports the number of times each pitfall appears in the analyzed ontology. Examples of pitfalls are: (a) creating synonyms as classes (when several classes whose identifiers are synonyms are created and defined as equivalent); (b) creating unconnected ontology elements (when some ontology elements are created that bear no relation to the rest of the ontology); (c) defining wrong inverse relationships (when two relationships are defined as inverse relations and are not necessarily so); (d) missing disjointness ${ }^{50}$ (when axioms between classes or between properties that should be defined as disjoint are omitted from the ontology); and (e) missing domain or range in properties (when the ontology includes relationships and/or attributes without domain or range (or either)). This criterion should be measured using a discrete attribute based on the number of pitfalls present in the candidate ontology. Thus, the attribute range would be $[0,21]$.

Purpose of use (Purpose Use) refers to the (subjective) idea that the candidate ontology's end use can affect the perception of the ontology's reliability. A discrete attribute with three possible values was established to measure this criterion:

- low, when the candidate ontology was developed for internal and/or academic use;

- medium, when the candidate ontology was developed as a part of a national research project and

- high, when the candidate ontology was developed as a part of a European or international research project.

Finally, popularity refers to whether there are any projects, applications or ontologies reusing the candidate ontology. ${ }^{51}$ This criterion can be measured using the following discrete values:

- low, when the candidate ontology has been reused zero times;

- medium, when the candidate ontology has been reused between 1 and 3 times;

- high, when the candidate ontology has been reused between 4 and 7 times and

- very high, when the candidate ontology has been reused 8 or more times.

Note that there is no need to set apart projects, applications and ontologies that reuse the candidate ontology to analyze this criterion.

\subsection{Candidate ontologies and their performances}

Nowadays, the sports domain is receiving growing attention from broadcasters and producers, sponsors and viewers. People are continuously consuming multimedia contents in different formats and from different sources using Google, Flickr, Picasa, YouTube, and so on. In most cases, people want to quickly locate multimedia contents giving a natural language description of what they want (e.g., videos about the 
London Olympic Games, or videos about the 2010 FIFA World Cup featuring Iker Casillas and Andrés Iniesta). To do this, multimedia contents need to be semantically described for interpretation by both human agents (users) and machine agents (computers). In particular, multimedia contents about sports can be described semantically using sport ontologies.

In this respect, an ontology network called $M 3^{\circ}$ is being developed as part of Buscamedia project. ${ }^{\mathrm{h}}$ This ontology network covers three perspectives: it should be a multimedia, multilingual and multidomain ontology network. Within the multidomain perspective, we have developed a sports ontology network following the NeOn Methodology. In this paper, we describe the reuse process performed during the development of such a sports ontology.

First we searched for ontologies in the sports domain using two semantic search engines (Swoogle ${ }^{i}$ and Watson ${ }^{j}$ ) and a general-purpose search engine (Google) according to the methodological guidelines for reusing domain ontologies presented in Sec. 2. Second, we examined these ontologies in depth to analyze their scope, purpose, and functional and nonfunctional requirements against the specification for the new sports ontology to be developed. Examples of functional requirements that the new sports ontology should cover are 'what kinds of sport should it represent?', 'what types of football competition are there?', and 'how is a football match divided?'.

As a result of carrying out the domain ontology assessment activity, we have obtained the following nine candidate sport ontologies:

- Athlete ontology models the athletics domain, including concepts like athletes and several Olympic sports competitions. It is implemented in $O W L$ and available at http://www.mindswap.org/2004/athlete.owl.

- Baseball ontology describes baseball teams, players, games and pitches, catches, etc. It is available at http://www.daml.org/2001/08/baseball/baseball-ont and implemented in $O W L$.

- Olympic Sports Ontology (OSO) models the Olympic Games as well as different Olympic sports, which are divided into summer and winter sports. The ontology also lists medal winners. It is implemented in $O W L$ and available at http://swat. cse.lehigh.edu/resources/onto/olympics.owl.

- OntoSem ontology is a general-purpose ontology that describes different objects, events and properties as a model of the world. This ontology describes different sports, such as soccer, basketball and volleyball. It is implemented in $O W L$ and available at http://morpheus.cs.umbc.edu/aks1/ontosem.owl.

ghttp://mayor2.dia.fi.upm.es/oeg-upm/index.php/en/ontologies/224-buscamedia-ontologies-m3.

$\mathrm{h}_{\mathrm{http}: / / \text { www.cenitbuscamedia.es/. }}$

i http://swoogle.umbc.edu/.

j http://kmi-web05.open.ac.uk/WatsonWUI/. 
- Rissen ontology describes the world of football, including concepts such as competition, round, match, goal and team. It is implemented in $O W L$ and available at http://www.r4isstatic.com/linkeddata/ontologies/football/football.owl.

- Soccer ontology describes the basic concepts of soccer. The ontology defines actions during a match (player action, spectator action), types of players (central defender, goal keeper, etc.) or types of pass (chip pass, penetrating pass, push pass, etc.) and so on. It is implemented in $D A M L+O I L$ and available at http://www. atl.lmco.com/projects/ontology/ontologies/basketball_soccer/soccer.daml.

- Soccer Tsinakari ontology models agents, objects, events, places, time points and periods involved in soccer games. It is available directly from the author (Chrisa Tsinaraki) and implemented in $O W L$.

- SoccerV2 ontology describes most of concepts that are specific to soccer: players, rules, field, supporters, actions, etc. It is used to annotate videos in order to produce personalized summaries of soccer matches. It is implemented in it DAM $L+O I L$ and available at http://www.lgi2p.ema.fr/ ranwezs/ontologies/soccerV2.0.daml.

- UNSPSC (United Nations Standard Products and Services Code) ontology represents the Electronic Commerce Code Management Association ${ }^{k}$ UNSPSC code definition. This ontology includes an equipment-based taxonomy of sports. It is available at http://www.cs.vu.nl/ mcaklein/unspsc/unspsc84-title.rdfs and implemented in $R D F(S)$.

These candidate sports ontologies were analyzed with respect to the set of criteria and measurement attributes defined in Sec. 4.1 as part of the domain ontology selection activity. Table 2 shows the sports ontology performances for the 10 criteria under consideration. Note that DMs were allowed to use performance intervals (continuous attribute) or several discrete performances (discrete attribute) to enter imprecise performances. Table 2 does not include reuse cost because the nine candidate ontologies under consideration were available for free. Thus, this attribute was omitted from the analysis.

We also accounted for missing performances for ontology evaluation. The performances of two ontologies, OntoSem and UNSPSC, for this attribute were unknown. ' Consequently, the attribute range $([0,21])$ was considered as a missing performance, as explained in Ref. 52 .

\subsection{Quantification of DM'S preferences}

Quantifying preferences involves assessing single attribute utilities that represent DM preferences concerning the possible ontology performances. We accounted for imprecise information in the assessment process. This led to classes of

\footnotetext{
$\mathrm{k}_{\mathrm{h}} \mathrm{htp} / /$ www.eccma.org/.

${ }^{1}$ OntoSem and UNSPSC are not implemented using the standard RDF understood by JENA, which is the Java framework for building semantic web applications that supports OOPS!. For this reason, OOPS! was not able to evaluate such ontologies.
} 
Table 2. Sports ontology performances.

\begin{tabular}{lccccccccc}
\hline & Athlete & Baseball & OSO & OntoSem & Rissen & Soccer & Tsinaraki & SoccerV2 & UNSPSC \\
\hline Document A/Q & Low & Medium & Low & Medium & Low & Low & Low & Medium & Medium \\
Nam. Underst & 1 & 1 & 1 & 0 & 1 & 1 & 0 & 1 & 0 \\
Code Documen & 0 & 0 & {$[35,45]$} & 0 & {$[80,90]$} & 0 & {$[65,75]$} & 0 & 0 \\
Patterns & 0 & 0 & 0 & 0 & 0 & 0 & CP & 0 & 0 \\
Know Extract & 0 & 0 & 0 & 0 & 0 & 0 & 1 & 0 & 0 \\
Naming Conv. & 1 & 1 & 0 & 0 & 0 & 0 & 1 & 0 & 0 \\
Imp Language & High & High & High & High & High & Medium & High & Medium & Medium \\
O Evaluation & 2 & 2 & 6 & {$[0,21]$} & 2 & 0 & 0 & 0 & {$[0,21]$} \\
Purpose Rel & Low & Low & Low & High & Low & Low & Low & Medium & High \\
Popularity & Low & Medium & Low & Medium & Low & Medium & Low & Medium & Medium \\
\hline
\end{tabular}

utility functions for a continuous attribute, and utility intervals for each discrete attribute values.

Experts provided imprecise values to assess the component utility function for code documentation, see Fig. 2, whereas a decreasingly precise linear utility function was assigned to ontology evaluation.

Table 3 shows the imprecise component utilities identified for attributes on a discrete scale, excluding pattern conformance.

Regarding pattern conformance we considered that logical and content patterns are equally important and both more important than reasoning patterns. Table 4 shows the utilities for the discrete attribute values.

Quantifying preferences also involves eliciting weights that represent the relative importance of criteria. Weights were elicited along the branches of the hierarchy using a method based on trade-offs, ${ }^{19}$ accounting for imprecise responses to the probability questions put to DMs. This leads to intervals rather than precise weights. Figure 3 shows the average normalized weights across the objective hierarchy. Then, the attribute weights used in the multi-attribute additive utility model are assessed by multiplying the elicited weights in the path from the overall objective to the respective attributes.

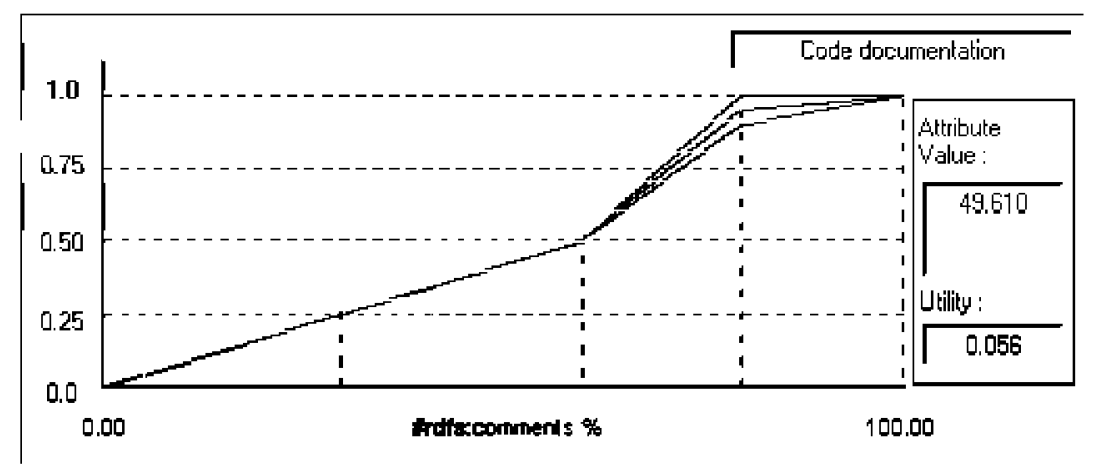

Fig. 2. Component utility function for code documentation. 
Table 3. Component utilities for discrete attributes.

\begin{tabular}{lcccccc}
\hline & Low & Medium & High & Very High & 0 & 1 \\
\hline A/Q Document & {$[0,0.2]$} & {$[0.3,0.6]$} & {$[0.7,1]$} & - & - & - \\
Nam. Underst & - & - & - & - & {$[0.0,0.2]$} & {$[0.3,1]$} \\
Know Extract & - & - & - & - & 0 & 1 \\
Naming Conv. & - & - & - & - & {$[0.0,0.3]$} & {$[0.4,1]$} \\
Imp Language & 0 & {$[0.4,0.6]$} & 1 & - & - & - \\
Purpose of use & {$[0,0.2]$} & {$[0.3,0.6]$} & {$[0.7,1]$} & - & - & - \\
Popularity & 0 & {$[0.3,0.4]$} & {$[0.7,0.8]$} & {$[0.9,1]$} & - & - \\
\hline
\end{tabular}

Table 4. Component utilities for pattern conformance.

\begin{tabular}{ccccccccc}
\hline & No patterns & $R P$ & $L P$ & $C P$ & $L P+R P$ & $C P+R P$ & $L P+C P$ & $L P+C P+R P$ \\
\hline Utility & 0.0 & {$[0.05,0.15]$} & {$[0.4,0.5]$} & {$[0.4,0.5]$} & {$[0.45,0.65]$} & {$[0.45,0.65]$} & {$[0.8,1.0]$} & 1.0 \\
\hline
\end{tabular}

Figure 4 numerically and graphically shows the average normalized weight and normalized weight intervals for the 10 attributes established in the lowest-level objectives for the selection of sport ontologies.

Documentation availability and quality is the most important attribute, followed by adequacy of knowledge extraction and adequacy of implementation language and ontology evaluation, respectively. This situation conforms to previous works ${ }^{3,46}$ where the most important criteria are related to the understandability effort, adequacy of knowledge extraction and ontology evaluation.

Note that the accuracy of weight elicitation methods decreases with the increase of the number of criteria. However, this is not the case for the problem under consideration since only 10 criteria were considered and weights were hierarchically

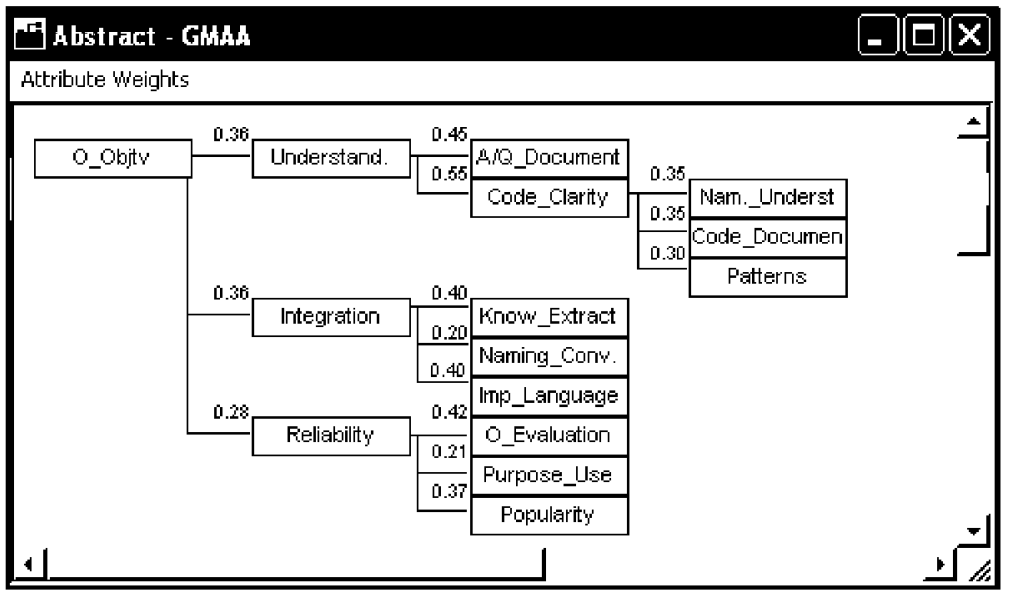

Fig. 3. Average weights representing the relative importance of criteria. 


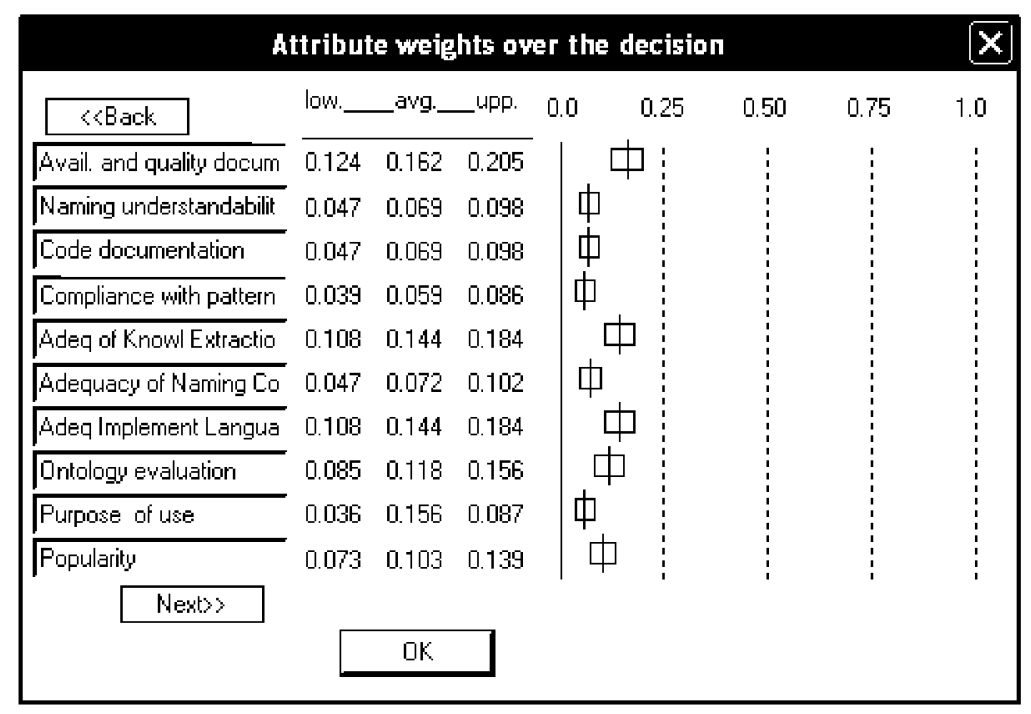

Fig. 4. Attribute weights.

elicited. In Ref. 53 a new method of determining the criteria weights is introduced, $F A R E$ (Factor Relationship), suitable for decision-making problems involving a high number of criteria.

\subsection{Evaluating ontologies and sensitivity analysis}

The additive model is considered a valid approach in most real decision-making problems for the reasons described in Refs. 54 and 55. The additive model is

$$
u\left(O_{i}\right)=\sum_{j=1}^{n} w_{j} u_{j}\left(x_{i j}\right),
$$

where $x_{i j}$ is the performance of ontology $O_{i}$ for attribute $X_{j} ; u_{j}\left(x_{i j}\right)$ is the utility associated with value $x_{i j}$, and $w_{j}$ are the weights of each attribute.

As we have accounted for imprecise information about the DM preferences and uncertainty about the ontology performances, the additive model is then used to assess average overall utilities and minimum and maximum overall utilities, see Fig. 5. A verage overall utilities (vertical line) are obtained by taking into account the mid-points of the performance intervals in the respective attributes (or the precise performance if applicable), their respective average component utilities and the average normalized attribute weights. To assess the minimum overall utilities, the system takes the lower end-points of the imprecise attribute weights, the lower endpoint of the performance intervals if the respective component utility function is increasing, or the upper end-point if it is decreasing (or the precise performance if applicable), and the lower utilities in the imprecise utilities corresponding to the above performances. 


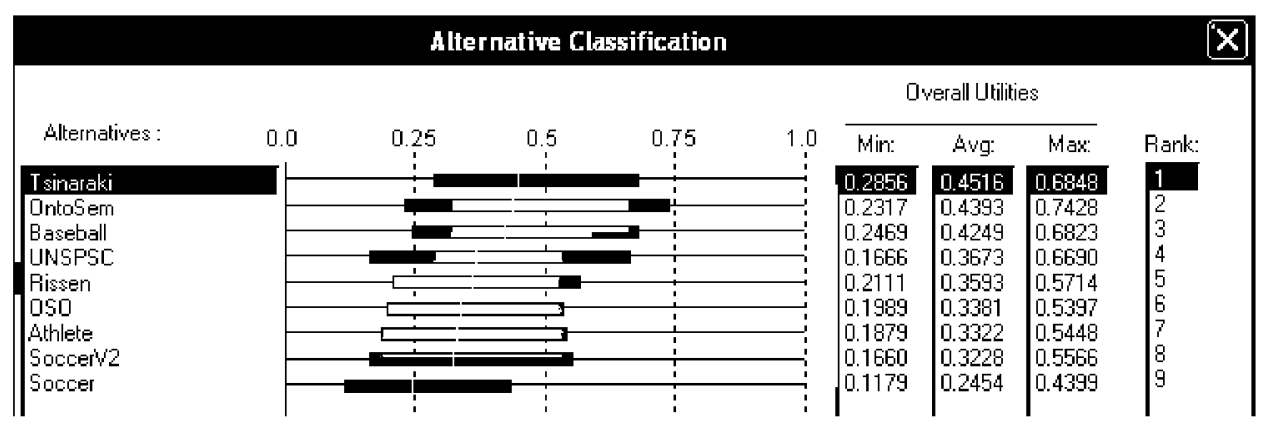

Fig. 5. Evaluation of sports ontologies.

The ranking of alternatives is based on the average overall utilities, and the minimum and maximum overall utilities provide further insight into the robustness of this ranking.

Note that according to the methodological guidelines on ontology reuse specified in NeOn Methodology, we have to select a subset of the top-ranked sports ontologies that simultaneously account for many of the CQs identified for the ontology under development.

Although Tsinaraki is the top-ranked sports ontology, its maximum utility is lower than for OntoSem and very similar to the value for Baseball and UNSPSC. Additionally, there is a big overlap of overall utility intervals of these ontologies. On other hand, the other sports ontologies appear to be inferior to the four topranked ontologies since their maximum overall utilities are clearly lower. Sensitivity analysis (SA) could provide further insight into the selection of the subset of sports ontologies.

The GMAA system provides tools to perform what is known as decision making with partial information. ${ }^{56,57}$ Decision making with partial information intends to take advantage of imprecise information about the decision-making problem.

Specifically, the GMAA system computes which of the nondominated alternatives are potentially optimal, ${ }^{58-60}$ that is, which are the top-ranked alternatives for at least one combination of imprecise parameters, i.e., weights and component utility functions. As Soccer is the only dominated ontology, it is the only alternative that can be discarded on the basis of this $\mathrm{SA}$, and further analysis is required to make a final selection.

The GMAA system also provides Monte Carlo simulation techniques. ${ }^{61,62}$ Monte Carlo simulation performs risk analysis by building models of possible results by substituting a range of values — a probability distribution — for any factor that is inherent uncertain. In the GMAA system, attribute weights are randomly assigned values taking into account the elicited weight intervals, see Fig. 4. While the simulation is running, the system computes several statistics about the rankings of each sports ontology (mode, minimum, maximum, mean, standard deviation and the 25th, 50th and 75 th percentiles) and outputs a multiple boxplot for the alternatives. 


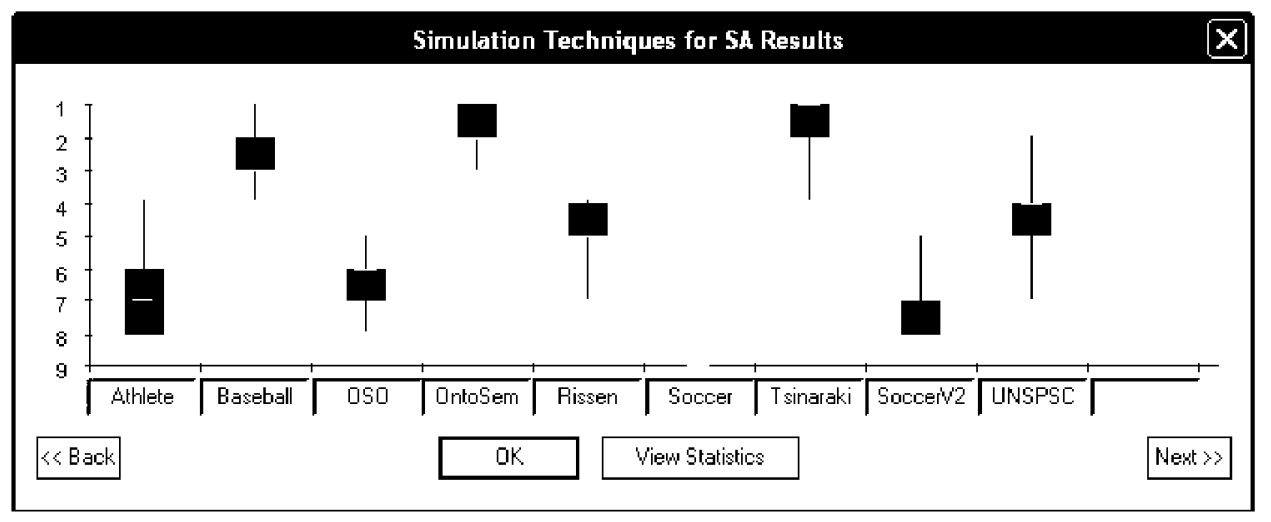

Fig. 6. Multiple boxplot.

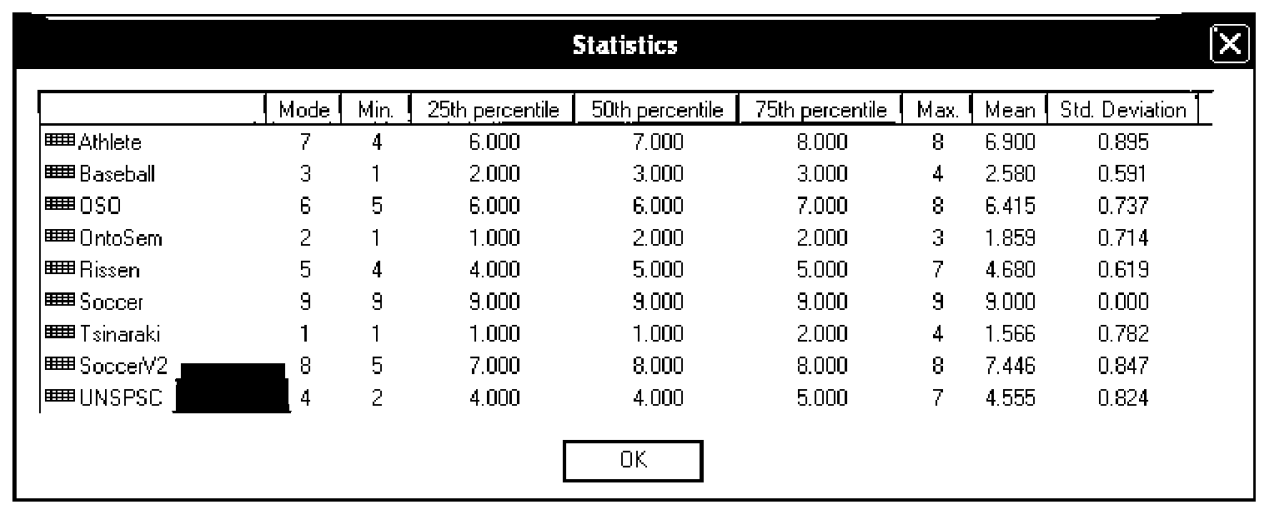

Fig. 7. Boxplot statistics.

Figure 6 shows the resulting multiple boxplot (the respective statistics are shown in Fig. 7). Looking at the box-plot corresponding to Athlete (Fig. 6) we find that the best rank for this ontology throughout the simulation is fourth, whereas its 25th percentile is sixth, its 75 th percentile is eighth and its worst percentile is eighth. Mode is seventh and the mean ranking is 6.9 (Fig. 4).

Only three sports ontologies ranked best across all 10,000 simulations: Baseball, OntoSem and Tsinaraki. These matches up with the results of the average overall utilities since the three top-ranked ontologies are the same as shown in Fig. 5. Their worst ranking is fourth, third and fourth, respectively. Also, the best ranking for UNSPSC is second but the worst is seventh, whereas the best ranking for the other ontologies is fourth. Consequently, we can conclude that the ranking is robust.

Taking into account the trade-off between number of selected ontologies and the percentage of covered requirements explained in Sec. 4, the set of selected ontologies are Baseball, OntoSem and Tsinaraki. This set of ontologies covers eight out of the 25 originally stated CQs. It is important to note that the next ontology in the ranking 
(UNSPSC) increases the number of CQs covered by only one. For this reason we decided not to include it in the final set of selected ontologies.

\section{Conclusion}

We have proposed a MAUT approach to deal with the selection of domain ontologies for reuse in the development a new ontology within the NeOn Methodology framework. As part of this approach, we have suggested the use of the GMAA decision support system to support the selection.

We have identified new attributes for measuring the performances of the candidate ontologies for the different criteria under consideration. Most are objective rather than subjective attributes and are easier for experts to measure. For instance, ontologies are evaluated using OOPS! to detect pitfalls. This is an advantage over earlier research on ontology reuse that tended to use subjective attributes.

The approach has been illustrated in a use case from the Buscamedia project concerning the development of an ontology in the sports domain. The GMAA has proved to be a very useful tool. It helps DMs to think about the problem in more depth, and accounts for imprecision in the inputs. This makes the process less stressful for experts and suitable for group decision making. Moreover, sensitivity analyses have proved to be especially useful for exploiting imprecise information about the input parameters to select a set of sports ontologies for reuse.

Note that the approach presented in this paper can be easily adapted for the selection of nonontological resources (e.g., thesauri, glossaries) and ontology design patterns. Preliminary adaptations have been performed as part of the Buscamedia project.

As a future research line, we propose to undertake a further analysis of the criteria and attributes used to measure the candidate ontology performances. For instance, the 21 common pitfalls detected using OOPS! may not be equally important, and the number of times each pitfall appears in the ontology is another factor to be taken into account. Thus, it might be worthwhile considering a weighted aggregation of the number of the different types of pitfalls, where it would be necessary to elicit the relative importance of the pitfalls. A simpler approach would be to build pitfall subsets that account for equally important pitfalls, eliciting the relative importance of each subset and counting the number of times pitfalls from each subset appear.

Additionally, taking advantage of the fact that the $O E G$ research group ${ }^{\mathrm{m}}$ is involved in many EU projects on ontology development and collaborates with numerous ontology engineering experts around the world, interviews and questionnaires could be administered to build a consensus on such attributes, i.e., to come up with a collection of criteria created with ontology community agreement.

Moreover, consensus could also be reached on weights representing the relative importance of criteria. Additionally, we have planned an experiment with ontology

m Ontology Engineering Group (OEG), http://www.oeg-upm.net. 
experts to contrast the results of our approach with the results of experts selecting the set of ontologies themselves.

Finally, we are also going to analyze the possibility of providing RDF-based annotations (as an OMV extension or similar approach) for the ontologies analyzed following the approach presented in this paper.

\section{Acknowledgments}

The paper was supported by Madrid Regional Government project S-2009/ESP1685, the Spanish Ministry of Education and Science project TIN 2008-06796-C0402, the Spanish Ministry of Science and Innovation project MTM2011-28983-C03-03 and the CENIT-E project Buscamedia (CEN-2009-2016), co-funded by the Centro de Desarrollo Tecnológico Industrial (CDTI). We would like to thank our project partners for their help, and especially Ghislain Atemezing, whose master thesis was the starting point for this cooperation.

\section{References}

1. R. Studer, V. R. Benjamins and D. Fensel, Knowledge engineering: Principles and methods, Data \& Knowledge Engineering 25 (1998) 161-197.

2. A. Gómez-Pérez, M. Fernández-López and O. Corcho, Ontological Engineering, Advanced Information and Knowledge Processing Series (Springer-Verlag, Berlin, 2003).

3. M. C. Suárez-Figueroa, NeOn methodology for building ontology networks: Specification, scheduling and reuse, Ph.D. Thesis, Universidad Politécnica de Madrid, Madrid (2010), available at http://oa.upm.es/3879/.

4. P. Haase, S. Rudolph, Y. Wang, S. Brockmans, R. Palma, J. Euzenat and M. d'Aquin, Networked ontology model, NeOn Deliverable D1.1.1 (2006).

5. M. Fernández-López, A. Gómez-Pérez and N. Juristo, METHONTOLOGY: From ontological art towards ontological engineering, Spring Symp. Ontological Engineering of AAAI (Stanford University, California, 1997), pp. 33-40.

6. M. Uschold, M. Healy, K. Williamson, P. Clark and S. Woods, Ontology reuse and application, in Proc. Int. Conf. Formal Ontology and Information Systems, Trento (1998).

7. H. S. Pinto and J. P. Martins, A methodology for ontology integration, in Proc. 1st Int. Conf. Knowledge Capture, Victoria (2001), pp. 131-138.

8. E. Paslaru and M. Mochol, Towards a reuse-oriented methodology for ontology engineering, in Proc. 7th Int. Conf. Terminology and Knowledge Engineering, Copenhagen (2005), pp. 175-187.

9. M. C. Suárez-Figueroa, A. Gómez-Pérez, E. Motta and A. Gangemi (eds.), Ontology Engineering in a Networked World (Springer, Berlin, 2012).

10. H. S. Pinto and J. P. Martins, A methodology for ontology integration, in Proc. 1st Int. Conf. Knowledge Capture (ACM, New York, 2001), pp. 131-138.

11. A. Lozano-Tello and A. Gómez-Pérez, ONTOMETRIC: A method to choose the appropriate ontology, Journal of Database Management 2 (2004), 1-18.

12. H. Alani and C. Brewster, Metrics for ranking ontologies, in Proc. 4 th Int. EON Workshop, 15th Int. WWW Conf., Edinburgh (2006).

13. J. Park, S. Oh and J. Ahn, Ontology selection ranking model for knowledge reuse, Expert Systems with Applications 38 (2011), 5133-5144. 
14. M. Martínez-Romero, J. M. Vázquez-Naya, J. Pereira and A. Pazos, A multi-criteria approach for automatic ontology recommendation using collective knowledge, in Recommender Systems for the Social Web, Intelligent Systems Reference Library, Vol. 32 (Springer-Verlag, Berlin, 2012), pp. 89-103.

15. M. C. Suárez-Figueroa, A. Gómez-Pérez and B. Villazón-Terrazas, How to write and use the Ontology Requirements Specification Document, in Proc. 8th Int. Conf. on Ontologies, DataBases and Applications of Semantics (ODBASE 2009), Vilamoura (2009).

16. A. Gómez-Pérez and A. Lozano-Tello, Applying ONTOMETRIC Method to Measure the Suitability of Ontologies, Business Systems Analysis with Ontologies, eds. P. Green and M. Rosemann (Idea Group Publishing, Hersey, 2005), pp. 249-269.

17. S. Peroni, E. Motta and M. d'Aquin, Identifying key concepts in an ontology, through the integration of cognitive principles with statistical and topological measures, in Proc. 3rd Asian Semantic Web Conf., Bangkok (2008), pp. 242-256.

18. R. Baeza-Yates and B. Ribeiro-Neto, Modern Information Retrieval (Addison Wesley, New York, 1999).

19. R. L. Keeney and H. Raiffa, Decisions with Multiple Objectives: Preferences and Value Tradeoffs (John Wiley \& Sons, New York, 1976).

20. W. Edwards, How to use multiattribute utility measurement for social decision-making, IEEE Transactions on Systems, Man, Cybernetics 7 (1977) 326-340.

21. L. Deng-Feng, Relative ratio method for multiple attribute decision making problems, International Journal of Information Technology 84 Decision Making 8 (2009) 289-311.

22. T. L. Saaty, The Analytic Hierarchy Process (McGraw-Hill, New York, 1980).

23. H. Eskandari and L. Rabelo, Handling uncertainty in the analytic hierarchy process: a stochastic approach, International Journal of Information Technology 8 Decision Making 6 (2007) 177-189.

24. B. Roy, Classement et choix en présence de points de vue multiples (la méthode ELECTRE), Revue Française d'Informatique et de Recherche Opérationnelle 8 (1968) $57-75$.

25. J. P. Brans, B. Mareschal and P. Vinke, How to select and how to rank projects: The PROMETHEE method, European Journal of Operational Research 24 (1986) 228-238.

26. B. Soylu, Integrating Promethee II with the Tchebycheff function for multicriteria decision making, International Journal of Information Technology 8 Decision Making 9 (2010) 525-545.

27. K. Supekar, Ch. Patel and Y. Lee, Characterizing quality of knowledge on semantic web, in Proc. AAAI Florida AI Research Symposium (FLAIRS-2004), Miami (2004).

28. R. Subhashini, J. Akilandeswari and V. Sinthuja, A review on ontology ranking algorithms, International Journal of Computer Applications 33 (2011) 6-11.

29. A. Esposito, M. Zappatore and L. Tarricone, Applying multi-criteria approaches to ontology ranking: A comparison with AKtiveRank, International Journal of Metadata Semantics Ontologies 7 (2012) 197-208.

30. A. Guitouni, J. M. Martel and P. Vincke, A framework to choose a discrete multicriterion aggregation procedure, Technical Report TR/SMG/2000-003, SMG, Universit Libre de Bruxelles (2000).

31. F. K. Linkov, A. Varhese, S. Jamil, T. P. Seager, G. Kiker and T. Bridges, Multicriteria decision analysis: A framework for structuring remedial decision at contaminated sites, in Comparative Risk Assessment and Environmental Decision Making, Nato Science Series: Earth and Environmental Sciences, Vol. IV (Springer, 2004), pp. 15-54.

32. F. K. Linkov, G. Satterstrom, G. Kiker, C. Batchelor, T. Bridges and E. Ferguson, From comparative risk assessment to multi-criteria decision analysis and adaptive 
management: Recent developments and applications, Environmental International 32 (2006) 1072-1093.

33. J. S. Dyer, Remarks on the analytic hierarchy process, Management Science 36 (1990) $249-258$.

34. J. E. Smith and D. von Winterfeldt, Decision analysis in management science, Management Science 50 (2004), 561-574.

35. M. Wang, S. J. Lin and Y. C. Lo, The Comparison Between MAUT and PROMETHEE, in Proc. 2010 IEEE Int. Industrial Engineering and Engineering Management (2010), pp. $753-757$.

36. V. Belton, Multiple criteria decision analysis - Practically the only way to choose, Operational Research Tutorial Papers (Operational Research Society, Birmingham, 1990), pp. 53-101.

37. R. T. Clemen, Making Hard Decisions (Duxbury Press, Beltmon, 1996).

38. C. W. Kirkwood, Strategic Decision Making: Multiobjective Decision Analysis with Spreadsheets (Duxbury Press, Beltmon, 1997).

39. A. Jiménez, S. Ríos-Insua and A. Mateos, A decision support system for multiattribute utility evaluation based on imprecise assignments, Decision Support System 36 (2003) $65-79$.

40. A. Jiménez, S. Ríos-Insua and A. Mateos, A generic multi-attribute analysis system, Computers and Operations Research 33 (2006) 1081-1101.

41. A. Jiménez, L. C. Rodríguez, S. Ríos-Insua and A. Mateos, Selection of segments to be sourced from low cost countries for a global industrial equipment manufacturer based on a multi-attribute decision support system, Research in Computing Science 17 (2005) 3-12.

42. A. Jiménez, A. Mateos, S. Ríos-Insua and L. C. Rodríguez, Contracting of cleaning services in a European underground transportation company with the aid of a DSS, Decision Support System 43 (2007) 1485-1498.

43. A. Jiménez, A. Mateos and A. Brhyn, Selecting intervention strategies against eutrophication and the drastic decrease in bird abundance in Ringkøbing Fjord, TOP 19 (2011) 448-463.

44. L. V. Utkin, Risk analysis under partial prior information and non-monotone utility functions, International Journal of Information Technology \& Decision Making 7(2007) $625-647$.

45. R. A. Aliev, W. Pedrycz and O. H. Huseynov, Decision theory with imprecise probabilities, International Journal of Information Technology 8 Decision Making 11 (2012) 271-306.

46. G. A. Atemezing, Analyzing and ranking multimedia ontologies for their reuse, M. Eng. Thesis Universidad Politécnica de Madrid, Madrid (2011), available at http:/ oa.upm.es/ $6368 /$.

47. A. Jiménez, M. C. Suárez-Figueroa, A. Mateos, M. Fernández-López and A. GómezPérez, A MAUT approach for reusing ontologies, in Proc. 28th IEEE Int. Conf. Data Engineering Workshops (IEEE, Washington, 2012), pp. 34-40.

48. A. Gómez-Pérez and M. D. Rojas-Amaya, Ontological Reengineering for Reuse, in Knowledge Acquisition, Modeling and Management: 11th European Workshop on Knowledge Acquisition, Modeling and Management, Lecture Notes in Computer Science, Vol. 1621 (Springer-Verlag, Berlin, 1999), pp. 139-156.

49. M. Poveda-Villalón, M. C. Suárez-Figueroa and A. Gómez-Pérez, Validating ontologies with OOPS!, in Knowledge Engineering and Knowledge Management, Lecture Notes in Computer Science Vol. 7603 (Springer-Verlag, Berlin, 2012), pp. 267-281. 
50. A. Gómez-Pérez, Ontology evaluation, in Handbook on Ontologies, International Handbooks on Information Systems, eds. S. Staab and R. Studer (Springer, Berlin, 2004), pp. 251-274.

51. A. Lozano-Tello, Métrica de idoneidad de ontologías, Ph.D. thesis, Universidad de Extremadura, Cáceres (2002).

52. A. Jiménez, S. Ríos-Insua and A. Mateos, Missing consequences in multi-attribute utility theory, OMEGA 37 (2009) 395-410.

53. R. Ginevicius, A new determining method for the criteria weights in multicriteria evaluation, International Journal of Information Technology \& Decision Making 10 (2011) 395-410.

54. H. Raiffa, The Art and Science of Negotiation (Harvard University Press, California, 1982).

55. T. J. Stewart, Robustness of additive value function method in MCDM, Journal of Multi-Criteria Decision Analysis 5 (1996) 301-309.

56. D. Ríos Insua, Sensitivity Analysis in Multi-objective Decision Making (Springer, New York, 1990).

57. D. Ríos and S. French, A framework for sensitivity analysis in discrete multi-objective decision-making, European Journal of Operational Research 54 (1991) 176-190.

58. Y. S. Eum, K. S. Park and S. H. Kim, Establishing dominance and potential optimality in multi-criteria analysis with imprecise weight and value, Computers 8 Operations Research 28 (2001) 397-409.

59. K. S. Lee, K. S. Park and S. H. Kim, Dominance, potential optimality, imprecise information and hierarchical structure in multicriteria analysis, Computers \& Operations Research 29 (2002) 1267-1281.

60. A. Mateos, S. Ríos-Insua and A. Jiménez, Dominance, potential optimality and alternative ranking in imprecise decision making, Journal of the Operational Research Society 58 (2007) 326-336.

61. A. Mateos, A. Jiménez and S. Ríos-Insua, Monte Carlo simulation techniques for group decision-making with incomplete information, European Journal of Operational Research 174 (2006) 1842-1864.

62. J. Butler, J. Jia and J. Dyer, Simulation techniques for the sensitivity analysis of multicriteria decision models, European Journal of Operational Research 103 (1997) 531-546. 